\title{
PREPL deficiency: delineation of the phenotype and development of a functional blood assay
}

\author{
Luc Régal, $\mathrm{MD}^{1,2,12}$, Emma Mårtensson, $\mathrm{PhD}^{3}$, Isabelle Maystadt, $\mathrm{MD}, \mathrm{PhD}^{4}$, \\ Nicol Voermans, $\mathrm{MD}, \mathrm{PhD}^{5}$, Damien Lederer, $\mathrm{MD}, \mathrm{PhD}^{4}$, Alberto Burlina, $\mathrm{MD}, \mathrm{PhD}^{6}$, \\ María Jesús Juan Fita, MD ${ }^{7}$ A. Jeannette M. Hoogeboom, MD ${ }^{8}$, Mia Olsson Engman, MD ${ }^{9}$, \\ Tess Hollemans ${ }^{2}$, Meyke Schouten, $\mathrm{MD}^{10}$, Sandra Meulemans ${ }^{1}$, Tord Jonson, $\mathrm{PhD}^{3}$, \\ Inge François, MD, PhD ${ }^{11}$, David Gil Ortega, MD, Erik-Jan Kamsteeg, $\mathrm{PhD}^{10}$ and \\ John W.M. Creemers, PhD ${ }^{1}$
}

\begin{abstract}
Purpose: PREPL deficiency causes neonatal hypotonia, ptosis, neonatal feeding difficulties, childhood obesity, xerostomia, and growth hormone deficiency. Different recessive contiguous gene deletion syndromes involving PREPL and a variable combination of SLC3A1 (hypotonia-cystinuria syndrome), CAMKMT (atypical hypotoniacystinuria syndrome), and PPM1B (2p21 deletion syndrome) have been described. In isolated PREPL deficiency, previously described only once, the absence of cystinuria complicates the diagnosis. Therefore, we developed a PREPL blood assay and further delineated the phenotype.

Methods: Clinical features of new subjects with PREPL deficiency were recorded. The presence of PREPL in lymphocytes and its reactivity with an activity-based probe were evaluated by western blot.

Results: Five subjects with isolated PREPL deficiency, three with hypotonia-cystinuria syndrome, and two with atypical hypotonia-
\end{abstract}

cystinuria syndrome had nine novel alleles. Their IQs ranged from 64 to 112. Adult neuromuscular signs included ptosis, nasal dysarthria, facial weakness, and variable proximal and neck flexor weakness. Autonomic features are prevalent. PREPL protein and reactivity were absent in lymphocytes from subjects with PREPL deficiency, but normal in the clinically similar Prader-Willi syndrome.

Conclusion: PREPL deficiency causes neuromuscular, autonomic, cognitive, endocrine, and dysmorphic clinical features. PREPL is not deficient in Prader-Willi syndrome. The novel blood test should facilitate the confirmation of PREPL deficiency.

Genet Med advance online publication 20 July 2017

Key Words: blood assay; hypotonia-cystinuria syndrome; neonatal hypotonia; Prader-Willi syndrome; PREPL

\section{INTRODUCTION}

Several autosomal recessive contiguous gene deletion syndromes have been described at $2 \mathrm{p} 21 .^{1}$ These deletions involve different combinations of four genes: PPM1B, SLC3A1, $P R E P L$, and CAMKMT (previously C2Orf34) (Figure 1f). $S L C 3 A 1$ is the gene for cystinuria type I, and when both alleles of SLC3A1 are deleted, cystinuria is present. This facilitates the diagnosis, as cystinuria can readily be detected by urinary amino acid analysis. ${ }^{2}$

The hypotonia-cystinuria syndrome, caused by deletions including PREPL and SLC3A1, has been described in 19 families, and is characterized by severe neonatal hypotonia which improves spontaneously, eyelid ptosis, facial weakness, and nasal dysarthria. Subjects with hypotonia-cystinuria syndrome often have a dry mouth with clumps of thickened saliva on the tongue (xerostomia). In addition, there are neonatal feeding problems, improving after infancy, and growth hormone deficiency, with a variable age at onset. Growth hormone therapy restores normal growth. Without dietary intervention, obesity develops in childhood. About half of the subjects with hypotonia-cystinuria syndrome need special education. Hypergonadotropic hypogonadism and delayed puberty occur. ${ }^{3-9}$

The syndrome caused by deletion of CAMKMT together with PREPL and SLC3A1, reported in one family, is called atypical hypotonia-cystinuria syndrome. ${ }^{10}$ Another family has been described where only PREPL and CAMKMT were deleted, without cystinuria. ${ }^{11}$ Mitochondrial dysfunction (complex IV) is a feature of these two syndromes, as well as mild to moderate intellectual disability, in addition to the neuroendocrine features of hypotonia-cystinuria syndrome. Electromyography showed chronic denervation and myotonic

\footnotetext{
${ }^{1}$ Laboratory of Biochemical Neuroendocrinology, Department of Human Genetics, University of Leuven, Leuven, Belgium; ${ }^{2}$ Pediatric Neurology and Metabolism, Department of Pediatrics, UZ Brussel, Brussels, Belgium; ${ }^{3}$ Department of Clinical Genetics, Region Skåne and Lund University, Lund, Sweden; ${ }^{4}$ Centre de Génétique Humaine, Institut de Pathologie et Génétique, Charleroi, Belgium; ${ }^{5}$ Department of Neurology, RadboudUMC Nijmegen, Nijmegen, The Netherlands; ${ }^{6}$ Division of Inherited Metabolic Diseases, Department of Pediatrics, University Hospital Padova, Padova, Italy; ${ }^{7}$ Unidad de Metabolopatías, Hospital Universitario Virgen de la Arrixaca, Murcia, Spain; ${ }^{8}$ Department of Clinical Genetics, Erasmus MC, University Medical Center Rotterdam, Rotterdam, The Netherlands; ${ }^{9}$ Department of Pediatrics, Blekinge Hospital, Karlskrona, Sweden;

${ }^{10}$ Department of Clinical Genetics, RadboudUMC Nijmegen, Nijmegen, The Netherlands; ${ }^{11}$ Department of Pediatric Endocrinology, UZ Leuven, Leuven, Belgium.

Correspondence: Luc Régal (Luc.regal@uzbrussel.be) or John WM Creemers (John.creemers@kuleuven.be)

${ }^{12}$ The first three authorscontributed equally to this work. 

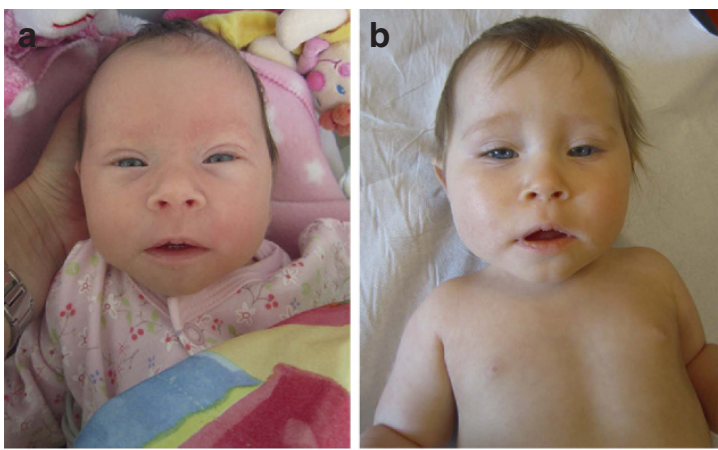

e

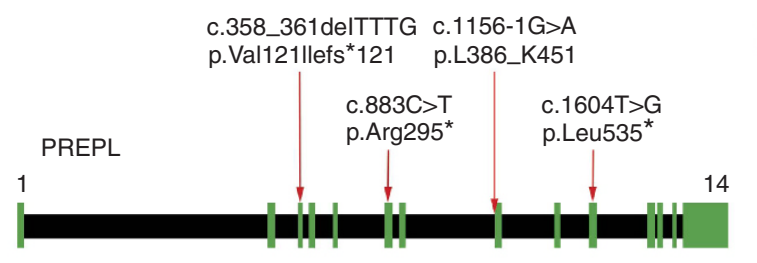

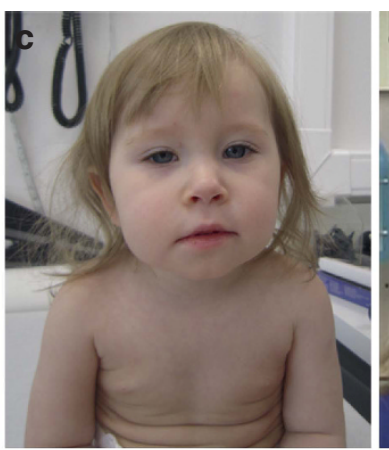

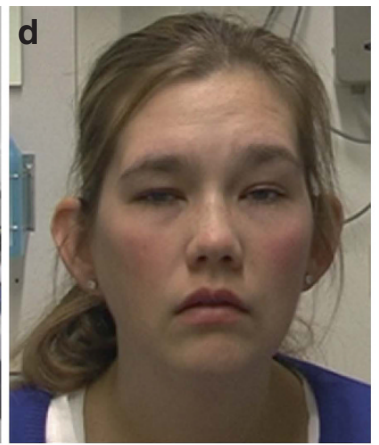

$\mathbf{f}$
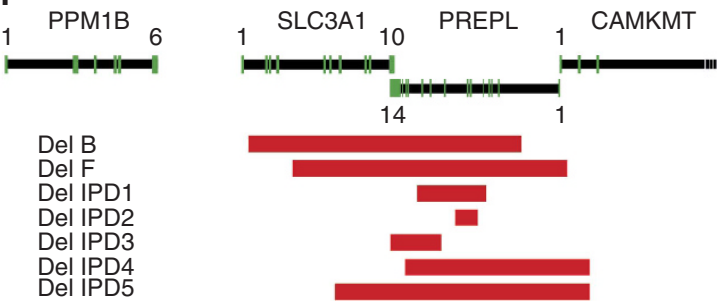

Figure 1 Facial features of isolated PREPL deficiency and overview of mutations. Subject IPD2 at the age of (a) 1 month, (b) 12 months, and (c) 20 months. Consent to publish photos was obtained. She has a poorly expressive face with ptosis and a tented upper lip. Notice the narrow bitemporal diameter, a short nose, and upslanting palpebral fissures. The facial dysmorphism becomes less obvious with age. (d) Subject IPD4 at the age of 27 years. She has poor facial expression and ptosis. Notice the narrow bitemporal diameter and prominent ears. e: mutations found by sequencing and their protein consequences. Numbering is according to NM_001171603.1. f: Deletions found by array-comparative genomic hybridization or qPCR. Deletions $\mathrm{B}^{3}$ and $\mathrm{F}^{10}$ have been reported before; the other mutations are novel.

discharges. ${ }^{10}$ Several undiagnosed hypotonic siblings died. Recently, growth restriction and mitochondrial dysfunction (complexes I, III, and IV) in the brain were demonstrated in a CAMKMT knockout mouse model. ${ }^{12}$

Additional deletion of PPM1B causes the most severe phenotype, the 2p21 deletion syndrome. Several children died, there were neonatal seizures, moderate to severe intellectual disability, and obvious mitochondrial dysfunction with lactic acidosis, ragged red fibers, and dysfunction of complexes I, III, IV, and V. ${ }^{13}$ This suggests both PPM1B and CAMKMT have a mitochondrial function.

Recently, we described the first subject with isolated PREPL deficiency. She was compound heterozygous for a deletion involving PREPL and SLC3A1 and a nonsense mutation in PREPL (p.Met270X). Her presentation was consistent with hypotonia-cystinuria syndrome, without cystinuria. There was transient improvement on pyridostigmine. Microelectrode studies of the neuromuscular junction at the age of 7 months demonstrated a neuromuscular junction defect, with decreased quantal size and quantal content, the latter caused by a decrease in vesicles available for release. The number of synaptic vesicles visualized at the neuromuscular junction and the distribution of postsynaptic acetylcholine receptors were normal. ${ }^{1}$

PREPL, or prolyl endopeptidase-like, encodes a cytoplasmic serine hydrolase structurally belonging to an oligopeptidase family. ${ }^{14}$ The substrate is not known. It reacts with a biotinylated fluorophosphonate (FP-biotin), ${ }^{15}$ an activity- based probe, if the configuration and accessibility of the catalytic triad (serine 470 , histidine 601, and aspartate 556) within the catalytic $\alpha / \beta$ hydrolase fold ${ }^{3}$ are intact. It is expressed ubiquitously, with highest expression in brain, muscle, and kidney. It partially colocalizes with the cytoskeleton and is also found associated with the Golgi membranes and in growth cones. ${ }^{16}$ PREPL was grouped among synaptic proteins using systems biology. ${ }^{17}$ It interacts with the $\mu 1 \mathrm{~A}$ subunit of the clathrin associated adaptor protein complex 1 and enhances the dissociation of this complex from target membranes. ${ }^{18}$ The endplate studies pointed to a defect in the late stages of synaptic vesicle exocytosis ${ }^{1}$ in isolated PREPL deficiency.

In almost all reported subjects with PREPL deficiency, detection of cystinuria or of minimally one PREPL deletion by array complete genome hybridization (array CGH) led to the diagnosis. Without these diagnostic clues, differential diagnosis with other causes of neonatal hypotonia is complicated. In practice this would involve whole-exome sequencing ${ }^{8,9}$ or a neuromuscular gene panel, including PREPL. As the course of PREPL deficiency is particular in the spontaneous improvement of the severe neonatal hypotonia and several manifestations can be treated, diagnosis is especially important. We therefore created a functional blood assay to detect PREPL protein and its reactivity with the activity-based probe FP-biotin. While developing this assay, we diagnosed several new subjects with PREPL deficiency, allowing us to broaden and better delineate the phenotype of PREPL deficiency. 


\section{Subjects}

\section{MATERIALS AND METHODS}

All procedures were performed after informed consent had been obtained according to the declaration of Helsinki. The evaluations were part of the study "PREPL in Health and Disease," ClinicalTrials.gov identifier: NCT02263781. The institutional review board of UZ Leuven approved the study. Physicians from various centers contacted L.R. after they identified subjects with PREPL deficiency. When possible, these subjects were clinically evaluated by L.R. (6 of 10) and/ or asked for a blood sample for the functional blood assay. A questionnaire was sent to the participating centers to complete. A narrative case report was also requested. In a second phase, the items in the questionnaire were adapted to include features repetitively described in the narrative case reports and sent again to the participating centers to be completed and verified. Details about the subjects are provided in Tables 1-3, Figure 1, and Supplementary Case Reports online.

IPD1

The subject was born as the first child of nonconsanguineous parents. She presented with severe neonatal hypotonia and feeding difficulties. Nasogastric tube feeding was started in the neonatal period. A percutaneous endoscopic gastrostomy tube was placed at the age of 6 months. From the age of 6 months tonus started to improve. She sat at 9 months. Feeding improved sufficiently to remove the gastrostomy at 6.5 years. She is currently gaining weight excessively. Cognitive and behavioral development is normal. At age 11 her heightgrowth velocity decreased and growth hormone deficiency was diagnosed. Growth hormone treatment was started. As puberty was delayed because of hypergonadotropic hypogonadism, estrogen supplementation was started at 14 years.

Molecular genetic and cytogenetic evaluation for PraderWilli syndrome, myotonic dystrophy type I, and spinal muscular atrophy were negative. Array CGH showed a homozygous deletion in PREPL. Both parents were heterozygous.

\section{IPD2}

This subject (Figure 1a-c) was born at 38 weeks by Cesarean section because of breech presentation. Her parents were nonconsanguineous. Pregnancy was complicated by intrauterine growth restriction. At clinical exam, she had bilateral ptosis, a tented upper lip, and moderate axial hypotonia. In addition to the dysmorphic features mentioned in Table 1, she had upslanting palpebral fissures and a short nose. Cranial, cardiac, and abdominal ultrasound were normal. Plasma creatine kinase was normal. She developed feeding difficulties with a poor sucking reflex.

After the age of 3 months, her appetite improved but swallowing liquids remained very difficult until the age of 13 months. Height evolved at -5 standard deviation score (SDS) and weight at -4.5 SDS. Head circumference evolved at -2 SDS. Maximal growth hormone $(2.07 \mathrm{ng} / \mathrm{ml})$ after glucagon injection indicated growth hormone deficiency.

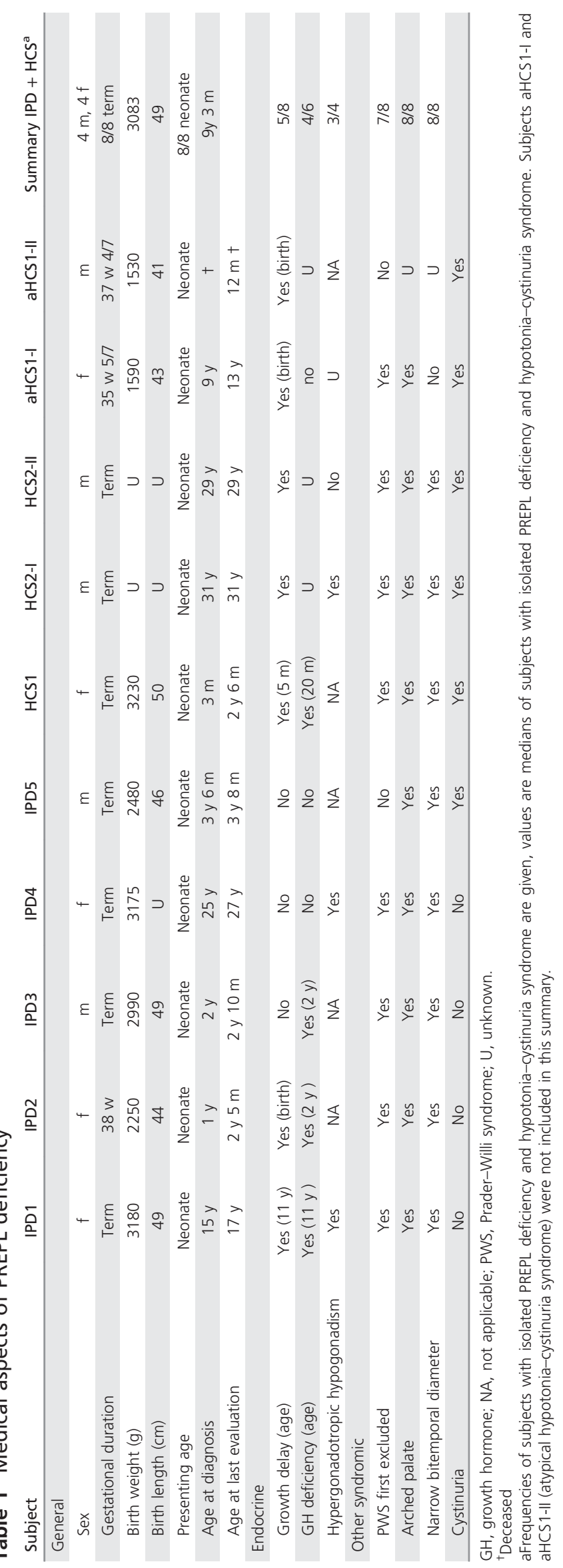




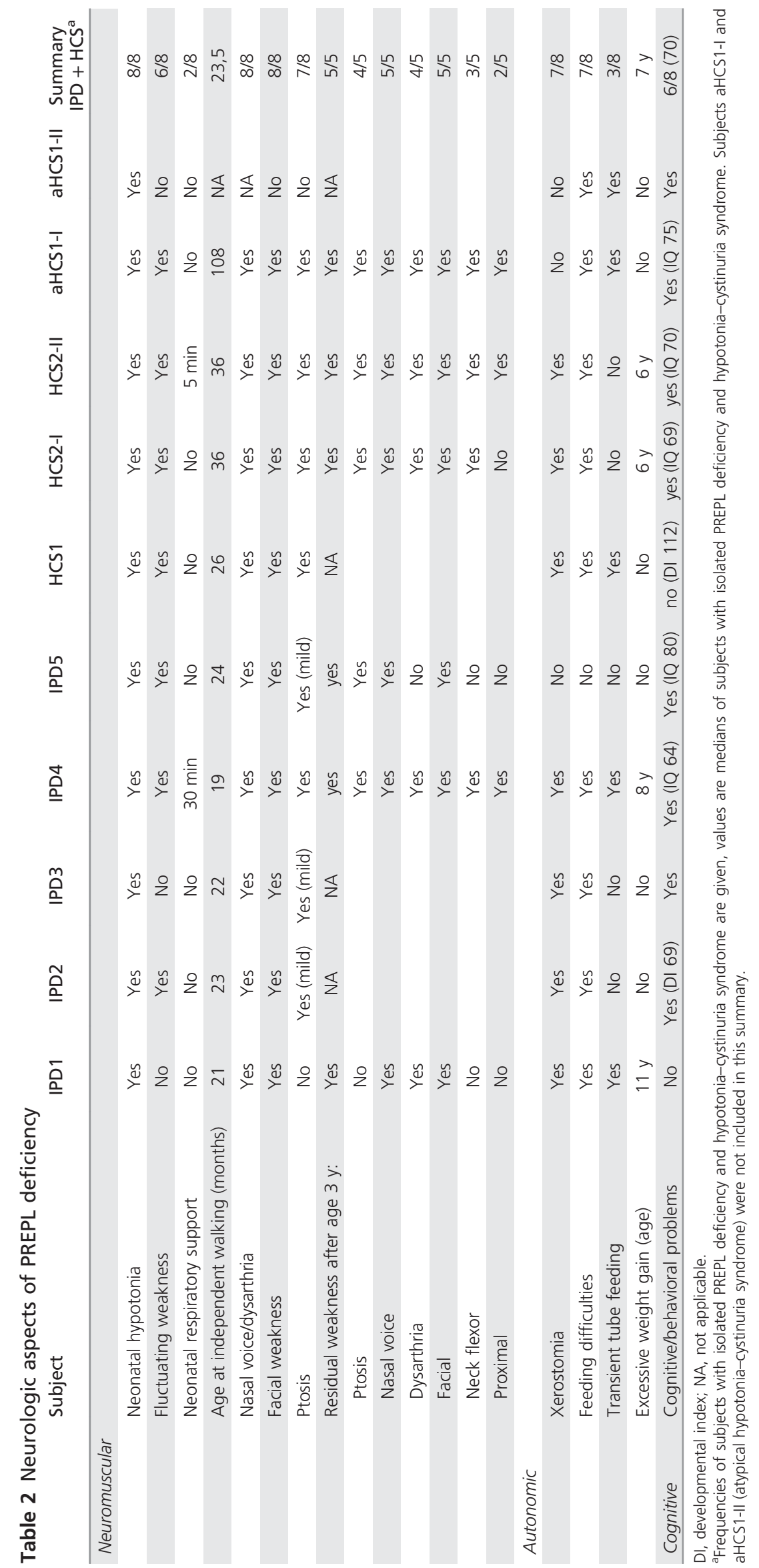


Table 3 Molecular genetics of the new subjects with PREPL deficiency

\begin{tabular}{|c|c|c|}
\hline Subject & Deletion & Mutation \\
\hline IPD1 & HOM (PREPL) 2p21(44559541-44571008) Hg19 (PREPL exon 10-5) & \\
\hline IPD2 & HET (PREPL) 2p21(44562458-44568223) Hg19 & HET (c.358_361delTTTG ; p.Val121llefs*121) \\
\hline IPD3 & HET (SLC3A1, PREPL) 2p21(44545015-44556286) Hg19 & HET (c.1604T > G ; p.Leu535*) \\
\hline IPD4 & HET (PREPL, CAMKMT) 2p21 (PREPL exon 12-CAMKMT exon 2) & HET (c.883 C > T; p.Arg295*) \\
\hline IPD5 & HET (SLC3A1, PREPL, CAMKMT) (SLC3A1 exon 7-CAMKMT exon 2) & HET (c.1156-1 G > A; p.L386_K451del) \\
\hline HCS1 & HOM Del B & \\
\hline aHCS1-I & HOM Del F & \\
\hline aHCS1-\| & HOM Del F & \\
\hline
\end{tabular}

The reference sequence was NM_001171603.1.

HET, heterozygous; HOM, homozygous.

She sat at 11 months and crawled at 14 months. At the age of 29 months, she showed small joint hypermobility, and persistent mild to moderate axial hypotonia. She could pronounce a few words. Developmental index (Bayley Scales of Infant Development-III-FR) was 69. Brain MRI and MRI spectroscopy at 9 months were normal.

Molecular genetic evaluation for mosaic trisomy 21, Prader-Willi syndrome, myotonic dystrophy type 1 , and spinal muscular atrophy was negative. Chromosome 7 uniparental disomy and hypomethylation of paternal $11 \mathrm{p} 15$ (Silver-Russell syndrome) were excluded.

Array CGH revealed a paternally inherited 6-kb microdeletion within PREPL. Sequencing of PREPL revealed a maternally inherited 4-bp deletion.

\section{IPD3}

The subject was born by Cesarean section from nonconsanguineous parents. There was profound neonatal hypotonia with absent head control. In addition to the dysmorphic features mentioned in Table 1, he had prominent ears and a short nose. He was breastfed for 1 week but was then switched to syringe feeding because of feeding difficulties. Feeding difficulties started to improve at the age of 18 months, and at 29 months a full meal lasted 15 minutes. He showed persistent failure to thrive. Weight followed the -3.2 SDS from the age of 3 months. Height and head circumference are on the 25th percentile. He has mild dysmorphic features. Development is delayed (uses two words at 34 months, but understands well). He has had a flat smile and ptosis since the neonatal period. He sat unsupported at 11 months. At the age of 34 months, he had a myopathic face, eyelid ptosis, nasal dysarthria, and mild proximal weakness. Neck flexion was paretic.

Cardiac, renal, and cranial ultrasound were normal at the age of 3 months.

Array CGH revealed a heterozygous deletion in PREPL and sequencing of the remaining allele of PREPL revealed a nonsense mutation.

\section{IPD4}

The subject's parents were nonconsanguineous. The neonatal period was characterized by severe hypotonia, respiratory insufficiency, and feeding problems with a poor sucking reflex. She received tube feeding for 4 months and afterwards was spoon-fed. She had gastro-esophageal reflux. Muscle force started to improve around 1 year of age. Her weight first evolved under the third percentile, but at the age of 8 years, she developed overweight. She underwent surgery for strabism. At the age of 12 years, her growth spontaneously accelerated, and she currently measures $170 \mathrm{~cm}$. Language acquisition was delayed. She received special education but is employed. She performed less well than her peers in physical education, and still becomes easily tired. There is a decrease in muscle strength during intercurrent infections and after physical exertion. She underwent bariatric surgery at the age of 24 years. At the last evaluation, she had a myopathic face, ptosis, nasal dysarthria, paresis of neck flexion, and milder paresis of deltoids, biceps, psoas, and hamstrings. Distal force was normal. She had fatigable diplopia. In addition to the dysmorphic features mentioned in Table 1, she had prominent ears and oligodentia (Figure 1d).

Nerve conduction studies with repetitive stimulation, electromyography, and single-fiber electromyography (orbicularis oculi) were normal at the age of 25 years. Muscle biopsy (at 24 years) showed rare cores (RYR1 sequencing normal), mild complex IV deficiency, but normal ATP production (mitochondrial DNA normal).

Molecular genetic evaluation for Prader-Willi syndrome was negative. A $250 \mathrm{~K}$ array CGH was normal. Whole-exome sequencing showed a heterozygous deletion involving PREPL and CAMKMT, and a heterozygous nonsense mutation in PREPL.

\section{IPD5}

This male subject was born after a pregnancy complicated by intrauterine growth retardation and oligohydramnion. He had neonatal hypotonia and poor feeding. Lactate was transiently elevated. Urinary amino acids showed cystinuria (cystine 
$758 \mathrm{mmol} / \mathrm{mol}$ creatinine (normal <36), lysine $3097 \mathrm{mmol} /$ mol creatinine (normal <182), arginine $2008 \mathrm{mmol} / \mathrm{mol}$ creatinine (normal <19). At 10 months he showed hypotonia, mild eyelid ptosis, and a tented upper lip. In addition to the dysmorphic features mentioned in Table 1, he had epicanthal folds and frontal bossing. He is hypermetropic. Renal ultrasound showed sludge in the right kidney.

qPCR showed a heterozygous deletion involving SLC3A1, $P R E P L$, and CAMKMT, inherited from the mother. On the other allele of $P R E P L$, a splice-site mutation, changing the universally conserved $G$ at position -1 of intron 8 to $A$, inherited from the father, was found. This abolishes the splice-acceptor site.

\section{Methods}

\section{Molecular genetics}

IPD1. Array CGH was done on Affymetrix CytoScan HD (Affymetrix, Santa Clara, CA), using equipment and protocols as recommended by the manufacturer. The deletion was further evaluated with qPCR.

IPD2 and IPD3. Array CGH was done on Agilent ISCA $180 \mathrm{~K}$ (Agilent, Santa Clara, CA). Sanger sequencing of the coding exons of PREPL was performed to evaluate the second allele in both subjects.

IPD4. A $250 \mathrm{~K}$ array CGH was performed. Whole-exome sequencing was done as described before. ${ }^{19,20}$

IPD5. qPCR for several exons of SLC3A1, PREPL, and CAMKMT was done on genomic DNA ${ }^{10}$ from both parents and IPD5. After a heterozygous deletion was found in IPD5 and his mother, primers were constructed to evaluate deletions in the $3^{\prime}$ untranslated region of PREPL, but no region of homozygous deletion was found. Therefore the coding exons of the other allele of PREPL were analyzed with Sanger sequencing. ${ }^{1}$

HCS1. qPCR for different exons of SLC3A1, PREPL, and CAMKMT was performed, and afterwards breakpoint PCR for deletion $\mathrm{B}$ was done. ${ }^{3}$

HCS2-I and HCS2-II. Array CGH was performed on an Illumina Human Cyto 12-SNP (Illumina, San Diego, CA) genotyping array. Afterwards, breakpoint PCR for deletion B was done. ${ }^{3}$

aHCS1-I and aHCS1-II. qPCR for different exons of SLC3A1, PREPL, and CAMKMT was performed. ${ }^{10}$ Breakpoint PCR for deletion $\mathrm{F}$ was done. ${ }^{10}$ Because insufficient DNA was available from aHCS1-II to perform numerous PCR and qPCR experiments, qPCR primers were constructed for the junction fragment of deletion F. Primers were chosen to amplify a fragment containing the breakpoint. This allowed us to confirm deletion $\mathrm{F}$ and to evaluate the number of affected alleles at the same time. As a confirmation, qPCR for exon 14 of PREPL was performed.

\section{Functional PREPL assay}

For a detailed description, see Supplementary Methods.

Peripheral blood lymphocytes and monocytes were isolated with Ficoll Paque plus (GE Healthcare Bio-Sciences AB,
Uppsala, Sweden). Isolated cells were cultured for 5 days in the presence of the lectin phytohemagglutinin, to stimulate cell division and metabolism in the T cells, which are the blood cells with the highest expression of PREPL. This results in an amplification of the number of $\mathrm{T}$ cells and of the amount of protein per $\mathrm{T}$ cell, reducing the amount of blood needed for the assay. After culturing for 5 days, the cells were harvested and slowly frozen to enhance lysis. After several days, the cells were thawed and mechanically lysed. The lysate was centrifuged to remove residual cellular debris and the protein concentration was measured in the supernatant. The supernatant was diluted to contain 200$2000 \mu \mathrm{g}$ protein/ml, depending on the experiment (Figure 2). PREPL was immune-precipitated from the supernatant with a mouse polyclonal antihuman PREPL antibody (MaxPab B01, Tevu-Bio, Le-Perray-en-Yvelines, France) and Trueblot Anti-Mouse Ig IP beads (Rockland, Limerick, PA), following the instructions of the manufacturer with minor modifications. Next, the immunoprecipitate was incubated with FP-biotin, the activity-based probe, ${ }^{15}$ with or without inhibitor 8, a selective PREPL inhibitor. ${ }^{21}$ When PREPL reacts with FP-biotin, FP-biotin covalently binds to the catalytic site, resulting in a biotin-labeled PREPL molecule. After the reaction, the samples were denatured, separated by SDSPAGE, and blotted. Reaction of PREPL with FP-biotin was demonstrated by incubation of the blots with streptavidinHRP, using the strong affinity of streptavidin for biotin. After the chemiluminescence reaction, the intensity of the band of PREPL reactivity with FP-biotin was quantified by densitometry with ImageJ. ${ }^{22}$ Next, the blots were stripped and incubated with another anti-PREPL antibody (Mouse Monoclonal IgG1 anti-PREPL antibody E-9, Santa Cruz Biotechnology, Dallas, TX) to detect the presence of PREPL (Figure 2a).

Several optimizations were carried out in later experiments to allow for quantification, to increase the signal, and to eliminate unspecific bands.

A quantified amount of bacterially expressed PREPL with a GST tag (PREPL-GST) ${ }^{14}$ was added to the diluted lymphocyte supernatant as a standard, to allow for quantification (Figure 2b-f, Supplementary Figure S1). This results in the addition of a higher migrating band of the standard (heavier owing to the GST tag) to the band of PREPL reactivity from the lymphocytes, in the same lane on the blot. Quantification is done by comparing the intensities of both bands with image-J. 22

To decrease proteolysis in the lymphocyte lysate and consequent signal loss, protease inhibitors at concentrations not inhibiting PREPL were added to the harvested lymphocytes before freezing Figure 2d-f, Supplementary Figure S1).

As the visualization of PREPL reactivity with FP-biotin in the assay depends on the interaction of streptavidin-HRP with biotin, unspecific bands can occur if an abundant naturally biotinylated protein would bind (in an unspecific way) to the immunoprecipitation beads. To avoid such unspecific bands, we added a preclearing step. In this step, High Capacity 


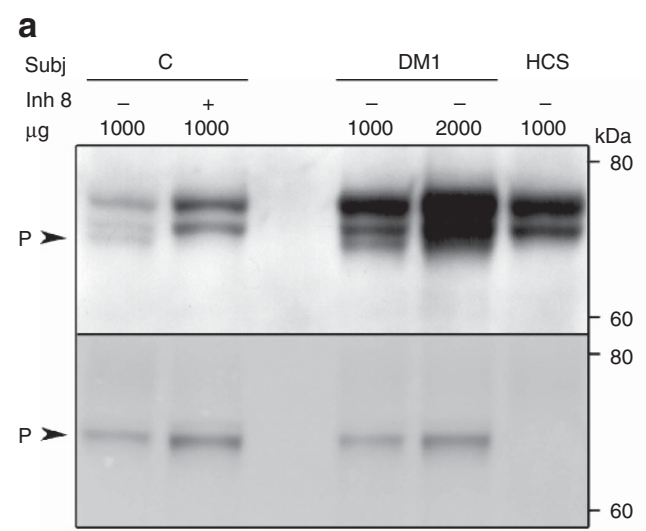

d

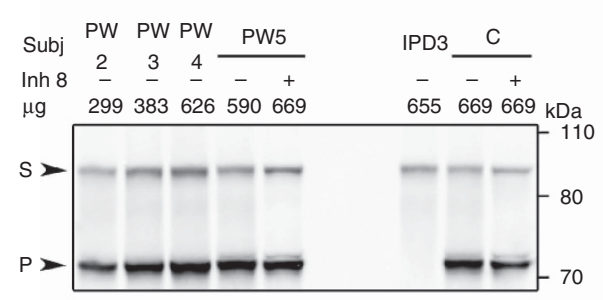

b

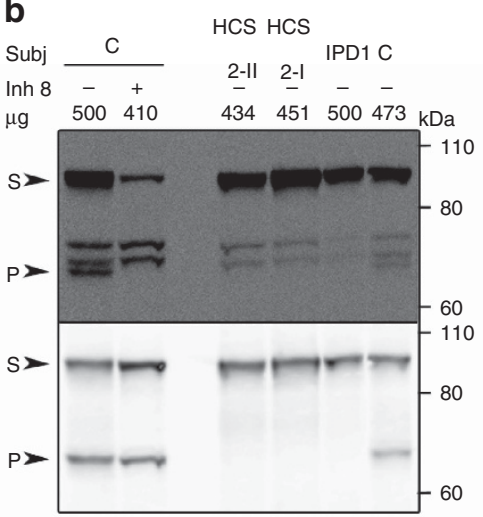

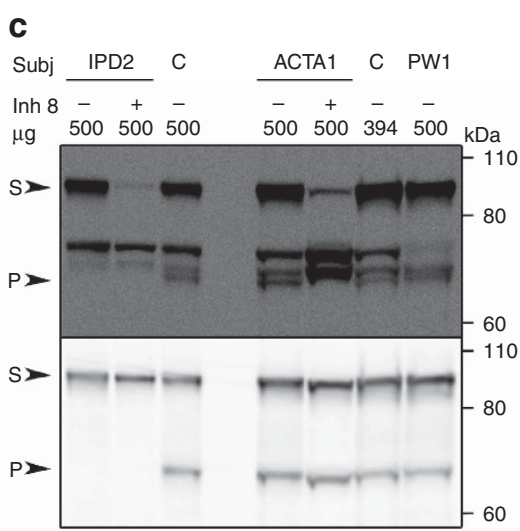

g

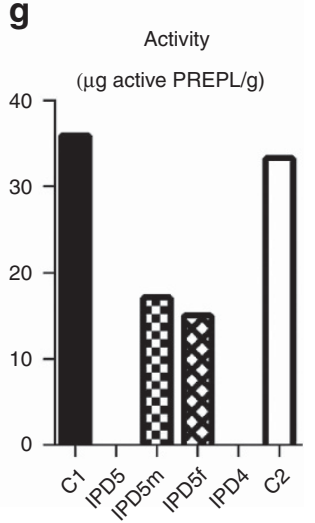

Figure 2 Optimization and performance of the PREPL assay. Western blots to demonstrate the presence of PREPL and its reactivity with FP-biotin. In the images with upper and lower panels, the upper panel represents the demonstration of PREPL reactivity with the activity-based probe FP-biotin and the lower panel represents the same blot after stripping and incubation with anti-PREPL antibody, demonstrating the presence of PREPL protein. (a) The control subject (C) shows three bands in the region of PREPL, of which only the lowest band disappears after inhibition of PREPL with inhibitor 8 (Inh 8). This band is present in a subject with congenital Steinert disease (DM1), but absent in a previously described subject with hypotonia-cystinuria syndrome $\left(\mathrm{HCS}\right.$, subject $\left.1-I^{3}\right)$. The lower panel shows the presence of PREPL in all conditions, except in the HCS sample. The amount of lymphocyte protein used is indicated in $\mu \mathrm{g}(\mu \mathrm{g})$. (b) and (c) $110 \mathrm{ng}$ PREPL-GST was added as a standard. Again, the specific lower band of PREPL after reaction with FP-biotin disappears after treatment with inhibitor 8 (upper panel), and subjects HCS 2-I and II, IPD1, and IPD2 show absence of PREPL reactivity with FP-biotin (upper panel) and PREPL protein (lower panel). Both PREPL after reaction with FP-biotin and PREPL protein are detectable in a subject with ACTA1-related nemaline myopathy (ACTA1) and a subject with Prader-Willi syndrome (PW1). (d) Inhibitors (EDTA and APMSF) were added to decrease variation by proteolysis. Eleven ng of PREPL-GST was added as a standard. The separation of the three bands in the region of PREPL in the activity-based assay was insufficient to identify PREPL and is therefore not shown. After stripping and incubation with anti-PREPL antibody, normal PREPL protein was detected in the control and four subjects with Prader-Willi syndrome, but PREPL is absent in IPD3. (e) and (f) Aspecific bands were removed by preclearing with streptavidin beads and changing the immunoprecipitation beads from Trueblot to sepharose $\mathrm{G}$ beads. Fifty-five nanograms of PREPL-GST was added as standard. This allows for unambiguous detection of PREPL reactivity with FP-biotin in the normal control, while both PREPL reactivity and PREPL protein are absent in IPD4 and IPD5. PREPL reactivity with FP-biotin and the amount of PREPL protein appear lower in the heterozygous parents (IPD5m and IPD5f) of IPD5. (g) Densitometric analysis of PREPL reaction with FP-biotin ( $\mu$ g active PREPL/g protein) in lymphocytes on western blot standardized to PREPL-GST for IPD4, IPD5, and IPD5 parents (IPD5m and IPD5f), as well as for the controls run at the same time. Despite different dilution, PREPL reactivity with FP-biotin of the controls is similar, while it is absent in IPD4 and IPD5, and about half that of the control in IPD5m and IPD5f (heterozygous). Photoshop was used to crop gel images. P arrowhead: PREPL; S arrowhead: PREPL-GST standard.

Streptavidin Resin (Thermo Scientific, Ghent, Belgium) was added to the diluted lymphocyte supernatant to bind and precipitate naturally biotinylated proteins. The resulting supernatant (without the bound biotinylated proteins) was then used for the immunoprecipitation of PREPL. Finally, the beads used for immunoprecipitation of PREPL were changed from Trueblot beads to Protein G Sepharose beads (GE Healthcare, Diegem, Belgium) because bead characteristics can influence the unspecific binding of interfering proteins (Figure 2e-f, Supplementary Figure S1).

\section{RESULTS}

The western blot for the reactivity with FP-biotin showed three bands in the expected region of PREPL and a higher band corresponding to PREPL-GST. Only the lowest band disappeared when inhibitor 8 , a specific inhibitor of PREPL, ${ }^{21}$ was added, indicating this band was specific for PREPL. The same band (but not the two higher bands) was absent in subjects with known hypotonia-cystinuria syndrome (Figure 2a), confirming it represents PREPL. PREPL reactivity with FP-biotin was absent in all tested novel 
subjects with PREPL deficiency, but present in healthy controls (Figure 2a-f).

The two unspecific bands were variably interfering with interpretation and quantification of PREPL reactivity with FP-biotin. Their size corresponded to the $\alpha$-subunits of methylcrotonyl-CoA carboxylase and propionyl-CoA carboxylase, two biotin-containing enzymes that are known to be induced by phytohemagglutinin..$^{23,24}$ This interference decreased incompletely after preclearing with streptavidin resin. With a rabbit PAb anti-propionyl-CoA carboxylase antibody (Bio-Connect, Huisen, The Netherlands), we showed that the lowest interfering band was indeed propionyl-CoA carboxylase (data not shown). Replacing the Trueblot beads by Protein G Sepharose beads in combination with preclearing with streptavidin resin eliminated the interfering bands (Figure 2e-f, Supplementary Figure S1). PREPL reactivity with FP-biotin of two carriers was approximately half of a normal control (Figure 2g).

After stripping and incubation with the E-9 anti-PREPL antibody to detect the presence of PREPL protein, there were no interfering bands, regardless of the preclear with streptavidin resin or the beads used for immunoprecipitation of PREPL. Only one higher band (corresponding to the standard, PREPL-GST) and one lower band (corresponding to the lymphocyte PREPL) were visible. As expected, both bands remained unchanged after treatment with inhibitor 8 . PREPL protein was absent in all subjects with PREPL deficiency but present in healthy controls.

Results in one subject with congenital myotonic dystrophy type 1, one subject with ACTA1-related nemaline myopathy, and five subjects with Prader-Willi syndrome were normal.

\section{DISCUSSION}

\section{Clinical findings}

After we published the report on the first subject with isolated PREPL deficiency, and demonstrated that it is a multisystem congenital myasthenic syndrome, ${ }^{1}$ here we present five new subjects with isolated PREPL deficiency, with a wide age range.

Severe neonatal hypotonia, nasal dysarthria, and neonatal feeding problems are constant findings in PREPL deficiency. Ptosis and facial weakness were observed in most subjects with PREPL deficiency, as well as fluctuations in weakness (Table 1 and 2). Subjects with hypotoniacystinuria syndrome or isolated PREPL deficiency walked at a median age of 23 months. The residual signs of neuromuscular involvement at adult age, except ptosis, are facial weakness, nasal dysarthria, and paretic neck flexion. Proximal weakness (arm abduction, hip flexion) after the age of 3 years was seen in two of five subjects with hypotoniacystinuria syndrome or isolated PREPL deficiency.

We present the first IQ data in PREPL deficiency. The cognitive profile in isolated PREPL deficiency and hypotoniacystinuria syndrome ranges from mild intellectual disability to normal intelligence. The median IQ was 70. It is unclear how this evolves over time.
Mild dysmorphic features, including an arched palate and narrow bitemporal diameter, were present in all subjects with PREPL deficiency. Growth hormone deficiency was detected in four subjects and age at onset ranged from 20 months to 11 years. Considering the absence of hypergonadotropic hypogonadism in isolated cystinuria and its frequent occurrence both in hypotonia-cystinuria syndrome and in isolated PREPL deficiency, we can also attribute this symptom to deficiency of PREPL.

The diagnosis of PREPL deficiency was often years after clinical presentation. In two out of eight families, technical and interpretative issues with array CGH delayed detection of relatively large deletions. Ensuring good coverage of the PREPL region in the array $\mathrm{CGH}$, and avoiding consideration of deletions in this region as benign copy-number variants is essential.

IPD5 had cystinuria. Because there was a mutation of a universal splice donor base pair in PREPL, explaining the demonstrated PREPL deficiency together with the deletion on the other allele encompassing SLC3A1, PREPL, and $C A M K M T$, we categorized him among the subjects with isolated PREPL deficiency. The cystinuria is probably due to an additional mutation in SLC3A1 or SLC7A9.

The second family with atypical hypotonia-cystinuria syndrome, described in this paper, confirms the clinical picture observed in the first family. ${ }^{10}$ This syndrome is more severe than hypotonia-cystinuria syndrome, considering both developmental outcome and mortality.

\section{Pathophysiological mechanisms}

Previous studies have demonstrated a defect in neuromuscular transmission that explains the severe neonatal hypotonia in PREPL deficiency. ${ }^{1}$ The striking but incomplete improvement of this hypotonia is not well understood. Although the eyelid ptosis fits well with the myasthenic signs, the normal single-fiber electromyography findings in the orbicularis oculi of IPD4 and in the frontal muscle of two previously reported subjects with hypotonia-cystinuria syndrome ${ }^{2}$ with persisting ptosis contradict this. We consider it more probable that ptosis is a sign of orthosympathetic involvement. Other signs indicating autonomous nervous system dysfunction are prevalent in PREPL deficiency, including slowing of gastric emptying, xerostomia, and tendency to obesity. ${ }^{1,3}$

How PREPL deficiency causes disease is unclear. PREPL was considered a peptidase, owing to its structural resemblance to prolyl endopeptidase (PREP). However, the activitybased probe FP-biotin was constructed as a general serine hydrolase inhibitor. ${ }^{15}$ Beside proteases, also lipases, phospholipases, esterases, cholinesterases, thioesterases, glycan hydrolases, and amidases belong to the serine hydrolase family. Despite many efforts, no peptide substrate for PREPL has been identified. ${ }^{3,14}$ In this context, it is worthwhile to note that two research groups showed around 50\% inhibition of PREPL (but not of PREP) by hexadecylfluorophosphonate (HDFP). ${ }^{25,26} \mathrm{HDFP}$, consisting of a fluorophosphonate group and a C16 chain resembling palmitate, was designed to 
identify serine hydrolases with palmitoylated proteins as a substrate. The amount of inhibition of PREPL by HDFP is comparable to that of several annotated enzymes with lipids as a substrate, including phospholipase A2 group $\mathrm{XV}$ (LYPLA3), acyl-CoA thioesterase 1 and 2 (ACOT1 and ACOT2), lysosomal acid lipase (LIPA) and $\alpha / \beta$-hydrolase domain containing protein 4 (ABHD4). These results raise the possibility that PREPL is not a peptidase but instead has a lipid substrate. This would be in agreement with the results of a search for a selective PREPL inhibitor, as the compounds found included molecules with an acyl group and esters, and needed a hydrophobic group to be active. ${ }^{21}$ Obviously, identification of a physiologic substrate would greatly facilitate our comprehension of the pathophysiological mechanisms in PREPL deficiency.

\section{Comparison with Prader-Willi syndrome}

In almost all subjects with PREPL deficiency, Prader-Willi syndrome was considered first. This reflects the striking qualitative resemblance between these two syndromes. Neonatal hypotonia with severe neonatal feeding problems, the spontaneous improvement, and the tendency to obesity in childhood are characteristic of both. Cognitive impairment, although more severe in Prader-Willi syndrome, is also common. Xerostomia and neuromuscular junction abnormalities ${ }^{27}$ are also features of Prader-Willi syndrome, as is growth hormone deficiency. ${ }^{28}$ Eyelid ptosis, however, is absent in Prader-Willi syndrome, and the severe behavioral problems in Prader-Willi syndrome are not seen in PREPL deficiency. As the long-term prognosis is clearly more favorable in isolated PREPL deficiency and hypotoniacystinuria syndrome, the differential diagnosis is crucial.

\section{Functional blood assay}

We established the first functional blood assay for PREPL deficiency. This novel assay, especially in its fully optimized form, reliably distinguishes between PREPL deficiency and controls. It can be done with $200 \mu \mathrm{g}$ of protein from stimulated lymphocytes. It gives normal results in subjects with Prader-Willi syndrome. Clinically, this allows differentiation between the two syndromes. Theoretically, this challenges the hypothesis that the resemblance between the two syndromes is caused by downregulation of PREPL in Prader-Willi syndrome. Any convergent pathophysiological mechanisms explaining the clinical similarity will be downstream of PREPL. The test was also normal in two subjects with other final neuromuscular diagnoses.

Although age-specific normal values have not been obtained yet, the reactivity of PREPL with FP-biotin in two heterozygotes was half that of a normal control. This suggests that the assay may also be useful to evaluate the functional relevance of missense mutations in PREPL that may be detected with the increasingly used next-generationsequencing techniques, probably in subjects with milder phenotypes.

\section{Conclusion}

Even though hypotonia-cystinuria syndrome and isolated PREPL deficiency are rare disorders, we suspect most subjects currently remain undiagnosed owing to lack of awareness about the condition. Groups where it would seem particularly appropriate to consider this diagnosis are subjects in whom a clinical suspicion of Prader-Willi Syndrome could not be confirmed by molecular genetics and subjects with severe neonatal hypotonia who improve remarkably, as in the group of subjects formerly diagnosed with "benign congenital hypotonia." 29

\section{SUPPLEMENTARY MATERIAL}

Supplementary material is linked to the online version of the paper at http://www.nature.com/gim

\section{ACKNOWLEDGMENTS}

L.R. received funding from FWO Vlaanderen (clinical doctoral fellowship) and from the Marie-Marguerite Delacroix fund. J.W. M.C. received funding from FWO Vlaanderen (G.0680.09), from European Community Seventh Framework Programme grant 223077, and from the Foundation for Prader-Willi Research.

\section{DISCLOSURE}

L.R. received funding from FWO Vlaanderen (clinical doctoral fellowship) and from the Marie-Marguerite Delacroix fund; he also received speakers' fees from Genzyme, and reimbursement of conference participation costs from Shire and Genzyme. To our knowledge, neither Genzyme nor Shire is active in projects concerning PREPL. J.W.M.C. received funding from FWO Vlaanderen (G.0680.09), from European Community Seventh Framework Programme grant 223077, and from the Prader-Willi research foundation. The other authors report no disclosures.

\section{REFERENCES}

1. Régal $L$, Shen XM, Selcen $D$, et al. PREPL deficiency with or without cystinuria causes a novel myasthenic syndrome. Neurology 2014;82: 1254-1260.

2. Boonen K, Régal L, Jaeken J, Creemers JW. PREPL, a prolyl endopeptidase-like enzyme by name only?-ILessons from patients. CNS Neurol Disord Drug Targets 2011;10:355-360.

3. Jaeken J, Martens K, Francois I, et al. Deletion of PREPL, a gene encoding a putative serine oligopeptidase, in patients with hypotonia-cystinuria syndrome. Am J Hum Genet 2006;78:38-51.

4. Eggermann T, Spengler $S$, Venghaus $A$, et al. 2 p21 Deletions in hypotonia-cystinuria syndrome. Eur J Med Genet 2012;55:561-563.

5. Martens K, Derua R, Meulemans S, et al. PREPL: a putative novel oligopeptidase propelled into the limelight. Biol Chem 2006;387:879-883.

6. Régal L, Aydin HI, Dieltjens AM, et al. Two novel deletions in hypotoniacystinuria syndrome. Mol Genet Metab 2012;107:614-616.

7. Clara R, Lowenthal A. Familial aminoaciduria with muscular hypotonia and dwarfism. Bull Acad R Med Belg 1966;6:577-611.

8. Wortmann SB, Koolen DA, Smeitink JA, van den Heuvel L, Rodenburg RJ. Whole exome sequencing of suspected mitochondrial patients in clinical practice. J Inherit Metab Dis 2015;38:437-443.

9. Legati A, Reyes A, Nasca A, et al. New genes and pathomechanisms in mitochondrial disorders unraveled by NGS technologies. Biochim Biophys Acta 2016;1857:1326-1335.

10. Chabrol B, Martens K, Meulemans S, et al. Deletion of C2orf34, PREPL and SLC3A1 causes atypical hypotonia-cystinuria syndrome. J Med Genet 2008;45:314-318.

11. Bartholdi D, Asadollahi R, Oneda B, et al. Further delineation of genotype-phenotype correlation in homozygous 2p21 deletion 
syndromes: first description of patients without cystinuria. Am J Med Genet A 2013;161A:1853-1859.

12. Haziza $S$, Magnani $R$, Lan $D$, et al. Calmodulin Methyltransferase is Required for Growth, Muscle Strength, Somatosensory Development and Brain Function. PLoS Genet 2015;11:e1005388.

13. Parvari R, Gonen Y, Alshafee I, Buriakovsky S, Regev K, Hershkovitz E. The 2 p21 deletion syndrome: characterization of the transcription content. Genomics 2005;86:195-211.

14. Szeltner Z, Alshafee I, Juhász T, Parvari R, Polgár L. The PREPL A protein, a new member of the prolyl oligopeptidase family, lacking catalytic activity. Cell Mol Life Sci 2005;62:2376-2381.

15. Liu Y, Patricelli MP, Cravatt BF. Activity-based protein profiling: the serine hydrolases. Proc Natl Acad Sci USA 1999;96:14694-14699.

16. Morawski $M$, Nuytens $K$, Juhasz $T$, et al. Cellular and ultra structural evidence for cytoskeletal localization of prolyl endopeptidase-like protein in neurons. Neuroscience 2013;242:128-139.

17. Oldham MC, Konopka G, Iwamoto K, et al. Functional organization of the transcriptome in human brain. Nat Neurosci 2008;11:1271-1282.

18. Radhakrishnan K, Baltes J, Creemers JW, Schu P. Trans-Golgi network morphology and sorting is regulated by prolyl-oligopeptidase-like protein PREPL and the AP-1 complex subunit $\mu 1 \mathrm{~A}$. J Cell Sci 2013;126(Pt 5): 1155-1163.

19. Neveling K, Feenstra I, Gilissen C, et al. A post-hoc comparison of the utility of sanger sequencing and exome sequencing for the diagnosis of heterogeneous diseases. Hum Mutat 2013;34:1721-1726.
20. de Ligt J, Boone PM, Pfundt R, et al. Detection of clinically relevant copy number variants with whole-exome sequencing. Hum Mutat 2013;34: $1439-1448$

21. Lone AM, Bachovchin DA, Westwood DB, et al. A substrate-free activitybased protein profiling screen for the discovery of selective PREPL inhibitors. J Am Chem Soc 2011;133:11665-11674.

22. Schneider CA, Rasband WS, Eliceiri KW. NIH Image to ImageJ: 25 years of image analysis. Nat Methods 2012;9:671-675.

23. Zempleni J, Mock DM. Utilization of biotin in proliferating human lymphocytes. J Nutr 2000;130(suppl 2S):335S-337S.

24. Kirkeby S, Moe D, Bøg-Hansen TC, van Noorden CJ. Biotin carboxylases in mitochondria and the cytosol from skeletal and cardiac muscle as detected by avidin binding. Histochemistry 1993;100:415-421.

25. Martin BR, Wang C, Adibekian A, Tully SE, Cravatt BF. Global profiling of dynamic protein palmitoylation. Nat Methods 2012;9:84-89.

26. Lin DT, Conibear E. ABHD17 proteins are novel protein depalmitoylases that regulate $\mathrm{N}$-Ras palmitate turnover and subcellular localization. Elife 2015;4:e11306.

27. Rajendran B, Young H, Tidswell T, Mansur A, Robb S, Pitt M. Abnormal neuromuscular transmission in infants with Prader-Willi syndrome. Neuromuscul Disord 2012;22:856-857.

28. Cassidy SB, Schwartz S, Miller JL, Driscoll DJ. Prader-Willi syndrome. Genet Med 2012;14:10-26.

29. Carboni P, Pisani F, Crescenzi A, Villani C. Congenital hypotonia with favorable outcome. Pediatr Neurol 2002;26:383-386. 